\title{
Validation of a mobility item bank for older patients in primary care
}

\author{
Julio Cabrero-García* ${ }^{*}$, Juan Diego Ramos-Pichardo', Carmen Luz Muñoz-Mendoza', \\ María José Cabañero-Martínez ${ }^{1}$, Lorena González-Llopis ${ }^{2}$ and Abilio Reig-Ferrer ${ }^{3}$
}

\begin{abstract}
Background: To develop and validate an item bank to measure mobility in older people in primary care and to analyse differential item functioning (DIF) and differential bundle functioning (DBF) by sex.

Methods: A pool of 48 mobility items was administered by interview to 593 older people attending primary health care practices. The pool contained four domains based on the International Classification of Functioning: changing and maintaining body position, carrying, lifting and pushing, walking and going up and down stairs.

Results: The Late Life Mobility item bank consisted of 35 items, and measured with a reliability of 0.90 or more across the full spectrum of mobility, except at the higher end of better functioning. No evidence was found of non-uniform DIF but uniform DIF was observed, mainly for items in the changing and maintaining body position and carrying, lifting and pushing domains. The walking domain did not display DBF, but the other three domains did, principally the carrying, lifting and pushing items.

Conclusions: During the design and validation of an item bank to measure mobility in older people, we found that strength (carrying, lifting and pushing) items formed a secondary dimension that produced DBF. More research is needed to determine how best to include strength items in a mobility measure, or whether it would be more appropriate to design separate measures for each construct.
\end{abstract}

Keywords: Mobility, Differential item functioning, Rasch analysis, Gender differences, Older people, Primary care, Item bank

\section{Background}

Physical function is a central component of health status and quality of life [1]. In addition to measuring physical function with fixed length scales such as the Health Assessment Questionnaire [2] or the subscale of physical functioning of the Medical Outcomes Study Short Form-36 (PF-10) [3], it can also be measured using item banks based on item response theory (IRT) models [4,5]. In some of these item banks, physical function is measured as a two-dimensional construct consisting of mobility and upper extremity function [6,7], although in others a unidimensional solution has been considered more appropriate $[8,9]$. Nevertheless, it is true that the latter is not sufficiently robust for certain health conditions [9]. The

\footnotetext{
* Correspondence: julio.cabrero@ua.es

${ }^{1}$ Department of Nursing, University of Alicante, Ctra. San Vicente s/n, San Vicente del Raspeig, Alicante 03015, Spain

Full list of author information is available at the end of the article
}

majority of these physical function measures are aimed at assessing health outcomes in patients with chronic diseases or in rehabilitation contexts [6-10]. However, there are no specific measures to assess physical function in community dwelling older people, with the exception of the Late-life Function and Disability Instrument $[11,12]$.

Measuring physical function - mainly mobility rather than upper extremity function - in older people is doubly useful as physical function is a strong predictor of disability, institutionalisation and death and is also a primary outcome, more proximal than disability, in longitudinal and clinical trials aimed at explaining or preventing disability $[13,14]$. Due to the scarcity and importance of late life mobility measures, the first of the two objectives of this paper is to present the development and validation of an item bank to measure mobility in community dwelling older people, using IRT methods. Items in the item bank were based on International Classification of Functioning, 
Disability and Health (ICF) mobility indicators [15]. Consequently, neither upper extremity function items nor disability (in activities of daily living) items were included.

In addition, significant gender differences in mobility have been observed, in the sense that women present a poorer function [16,17]. These differences are not uniform across the mobility domains, but are greater in the carrying, lifting and pushing domains than in the walking and moving domains [17-22]. However, psychometric studies analysing gender differential item functioning or DIF-namely, depending on construct level, whether the probability of responding to an item differs for the compared groups-have not yielded any relevant or systematic findings, except that most DIF effects are cancelled at the level of aggregate score $[8,9,12,23,24]$. For example, nine items in the physical function computerised adaptive testing version of the European Organisation for Research and Treatment of Cancer Quality of Life Questionnaire-Core 30 showed gender DIF, but DIF cancellation occurred because the DIF observed was in opposite directions: walking and moving items were more demanding for men whereas carrying, lifting and pushing items were more demanding for women [24].

However, although DIF cancellation can be secured in a fixed measure or even in an entire item bank, this is not the case in adaptive measures created from this latter $[23,25]$. In a standard DIF analysis, an internal criteriontotal score or an estimate based on total score-is used as a conditioning variable and then each item is individually studied for DIF [23]. However, it is also possible to study a bundle of items simultaneously rather than separately, and by analysing item bundles it becomes possible to test the DIF amplification hypotheses, i.e., whether items depending on a common secondary dimension have DIF effects, significant or nonsignificant, which accumulate at the level of item domain or bundle (differential bundle functioning or DBF) [26,27]. Accordingly, the second objective of this study was to examine whether mobility domains form secondary dimensions containing items that present DBF.

Therefore, the two objectives of this paper are to present the development and validation of an item pool to measure mobility in older people and to analyse differential item and bundle functioning across gender.

\section{Methods}

\section{Study population}

The data presented in this article have been taken from the baseline of a longitudinal study on mobility measures as predictors of adverse health outcomes. People considered eligible for participation in the study comprised those over 69 years old attending five primary health care centres in the Autonomous Region of Valencia (Spain). Those patients who produced more than three errors (four if they were illiterate) in the Short Portable
Mental Status Questionnaire [28], had serious communication problems or were considered too weak to participate in physical performance tests, were excluded. Sampling was consecutive: all eligible patients from one day of each week during the period November 2006 to October 2007 were selected. Of the 700 eligible patients, 593 gave informed consent and comprised the study sample. No statistically significant differences between participants and non-participants were observed for age or sex. The participants gave their informed consent and the study was approved by the corresponding authorities of the health centres involved.

\section{Measures}

\section{Late life mobility item bank (LLM-IB)}

A pool of 104 mobility items was selected from the literature and a panel of experts (two physicians, four nurses and three psychologists) assessed their relevance and suitability for older people, and also classified them into four domains based on three ICF categories of mobility: changing and maintaining body position (BP), carrying, lifting and pushing (CLP), walking (Walking) and going up and down stairs (UDS). Walking and UDS were considered separately and items relating to moving around using transportation were not included. The relevance of the activities included was also evaluated by three focus groups of older people. As a result of the above, 48 items were selected and their ease of understanding was assessed in 17 cognitive interviews. No items were eliminated, but modifications were made to various item statements. The item stem posed the question in terms of ability, in the present tense and made no reference to health, with a rating scale of four response categories: no difficulty, some difficulty, much difficulty and unable to do. Scores were scaled measuring mobility limitation: the higher the score, the worse the function.

\section{Other mobility measures}

PF-10 and the Short Physical Performance Battery (SPPB) were used as external criteria for the mobility item bank. PF-10 is a 10-item self-report measure based mainly on lower extremity mobility $[3,29]$. The SPPB battery objectively assesses physical function of the lower extremities. It consists of three tests: balance, gait speed and chair stand. It has demonstrated excellent reliability, predictive validity and sensitivity to clinically important change and has been recommended for objectively measuring mobility limitations $[14,30]$.

\section{Biodemographic, clinical and disability measures}

Biodemographic variables included body mass index $\left(\mathrm{kg} / \mathrm{m}^{2}\right)$, age, sex, education and living arrangements. Cognitive function was evaluated using the Short Portable Mental Status 
Questionnaire [28]. Symptoms of depression were evaluated with the Geriatric Depression Scale [31]. Morbidity was measured by the presence or absence of the following medical diagnoses: hypertension, rheumatoid arthritis, osteoarthritis, myocardial infarction, angina pectoris, congestive heart failure, diabetes, cancer, chronic pulmonary disease, stroke, hip fracture, Parkinson's disease, and claudication [32,33]. Finally, subjects were asked whether they needed the help of another person to complete any of the following activities: eating, toileting, bathing, dressing and transferring (ADL dependence).

\section{Procedure}

Measurements were collected at the primary health care centres, but not during the subject's medical appointment. The SPPB was administered by trained observers, who also recorded height and weight, morbidity was reported by the doctors caring for the patients who participated in the study and the other measures were completed in an interview situation, conducted by the same observers. Reliability of the mobility item pool and the SPPB was assessed in a pilot study. Using an interval of 15 days and a sample size of $n=62$, the intra-class correlation coefficient for intra-rater reliability was 0.90 for the entire item pool, with a range of $0.60-0.90$ for each of the items. Intra-class correlation coefficient for SPPB intra-rater reliability was $0.80(n=62)$ and for inter-rater reliability, $0.88(\mathrm{n}=30)$.

\section{Data analysis}

The main analyses consisted of examining DIF and DBF and calibrating the item pool using the Rasch rating scale model (RSM) [34]. Prior to this however, we performed a descriptive analysis of the items and examined the three assumptions common to IRT models: monotonicity, unidimensionality and local independency. Unidimensionality is also an assumption for standard DIF analysis. Since the unidimensionality of a measure in a population does not ensure its unidimensionality in subpopulations [35], this aspect was also analysed separately in the subsamples of women and men. DIF/DBF analysis was performed before calibrating the item pool to avoid confusing item DIF with item misfit

\section{IRT assumptions}

TestGraf [36] was used to analyse whether the items had a monotonic relation with the construct and if each response category had a maximum probability of being selected over a unique interval of the scale. TestGraf estimates and displays the characteristic response curves by means of the nonparametric regression method known as kernel smoothing. To examine the unidimensionality of the item pool, we tested confirmatory, single and bifactor models with factor analysis methods suitable for ordinal data, namely analysis of polychoric correlation matrices using a diagonally weighted least squares estimator $[4,37,38]$. We specified four group factors in the bifactor model, one for each mobility item pool domain. These analyses were performed for the entire sample and also for the male and female sub-samples. To measure goodness-of-fit of the models, we selected the Comparative Fit index (CFI), the Tucker Lewis Index (TLI), the root-mean-square error of approximation (RMSEA) and the standardised root mean square residual (SRMR) indices [4]. The cut-off values were as follows: 0.95 for TFI and CFI, 0.08 for RMSEA and 0.06 for SRMR $[4,39]$. For the bifactor models, we also estimated the proportion of variance explained by group and general factors, together with differences between common factor loadings for the single and bifactor models [38]. Moreover, residual correlations were calculated for the single factor models and $r>0.2$ was selected as the cut-off for determining the presence of local dependency [4]. LISREL was used for these analyses [37].

\section{Differential item and differential bundle functioning analysis by sex}

The simultaneous item bias test (SIBTEST) framework was used to assess DIF. SIBTEST is a nonparametric method which enables DIF to be tested both at item and item bundle levels [40]. An item bundle is a subset of substantively homogeneous or statistically dimensionally homogeneous items which measure a dimension secondary to the dominant dimension measured for the entire pool [40]. In this study, the bundles consisted of the four mobility item pool domains. SIBTEST permits formal statistical testing of item DIF and DBF, and a magnitude measure, $\beta$. The $\beta$ scale is the probability scale for single item analysis and the expected score scale for bundle analysis. Bundle $\beta$ is simply the sum of item $\beta$ for each of the bundle items [41].

Standard item DIF analysis uses an internal criterion, total score or a latent ability estimate, as a conditioning variable [35]. Since the conditioning variable should not have any items with significant DIF, a prior purification stage was implemented before the definitive item DIF analysis. The two types of DIF, uniform and nonuniform, were analysed: the Poly-SIBTEST (SIBTEST for ordinal data) was used to assess uniform DIF and the Crossing-SIBTEST for non-uniform DIF [42,43]. As only binary data can be analysed with the Crossing-SIBTEST, categories on the rating scale were combined as follows: no difficulty vs. the rest. Items were flagged for DIF if $\mathrm{P}<0.05$, using Bonferroni correction for multiple testing. We also conducted a sensitivity analysis of DIF: for uniform DIF we assessed differences between item locations produced in a Rasch RSM analysis, for each group, using 
t-tests; for non-uniform DIF, we used TestGraf to graphically examine the differences between the item response curves for each group.

To examine DBF, which is the cumulative effect of significant and nonsignificant item DIF across the item domain, we used two external criteria as conditioning variables, PF-10 and SPPB. Since PF-10 is a self-report measure, this criterion is the closest to the mobility item pool. However, SPPB, which is a mobility standard based on objective performance, can be useful for detecting pervasive DIF produced by self-report measures. Analysing DBF entails analysing item DIF, and therefore the results of the latter are also given.
IRT analysis: Rasch RSM

The item pool was calibrated using the Rasch RSM, the simplest Rasch model for polytomous items [44]. RSM allows items to vary in their level of difficulty but assumes that all items are equally discriminant and share the same rating scale structure [44]. Due to its more restrictive nature, it is robust for small or medium sized samples and is likely to provide more generalisable results [45]. In the RSM, response categories (K) are assigned intersection parameters ( $\mathrm{K}-1$ intersection parameters or thresholds) which are considered equal across items, and an item location is described by a single parameter that indicates the difficulty or ease of the item relative to category thresholds

\section{Table 1 Sample characteristics}

\begin{tabular}{|c|c|c|c|}
\hline & Sample $(n=593)$ & Male $(n=252)$ & Female $(n=341)$ \\
\hline \multicolumn{4}{|l|}{ Age } \\
\hline Mean (SD) & $76.53(4.81)$ & $76.73(4.78)$ & $76.39(4.84)$ \\
\hline Median & 76 & 76 & 76 \\
\hline Range & $70-98$ & $70-96$ & $70-98$ \\
\hline \multicolumn{4}{|l|}{ Gender (\%) } \\
\hline Female & 57.50 & & \\
\hline \multicolumn{4}{|l|}{ Education (\%) } \\
\hline Unable to read/write & 16.69 & 9.13 & 22.29 \\
\hline Able to read/write & 58.52 & 59.92 & 57.48 \\
\hline Primary & 16.86 & 19.44 & 14.97 \\
\hline Secondary or higher & 7.93 & 11.51 & 5.28 \\
\hline \multicolumn{4}{|l|}{ Living (\%) } \\
\hline Couple & 60.03 & 79.37 & 45.75 \\
\hline Family & 14.84 & 8.73 & 19.35 \\
\hline Alone & 25.13 & 11.90 & 34.90 \\
\hline \multicolumn{4}{|l|}{ Morbidity Index (\%) } \\
\hline $0-1$ & 21.92 & 29.37 & 16.42 \\
\hline 2 & 23.44 & 22.62 & 24.05 \\
\hline+2 & 54.64 & 48.01 & 59.53 \\
\hline Mean (SD) & $2.90(1.78)$ & $2.65(1.78)$ & $3.08(1.76)$ \\
\hline \multicolumn{4}{|l|}{ Body Mass Index } \\
\hline Mean (SD) & $29.12(4.28)$ & $28.14(3.81)$ & $29.85(4.46)$ \\
\hline \multicolumn{4}{|l|}{ Cognitive Status } \\
\hline SPMSQ: mean (SD) & $1.58(1.12)$ & $1.35(1.01)$ & $1.75(1.11)$ \\
\hline \multicolumn{4}{|l|}{ Functional Status } \\
\hline ADL dependence:\% & 6.41 & 3.57 & 8.50 \\
\hline SPPB: mean (SD) & $8.35(2.73)$ & $9.17(2.41)$ & $7.74(2.80)$ \\
\hline \multirow[t]{2}{*}{ PF-10: mean (SD) } & $67.90(26.35)$ & $76.38(22.45)$ & $61.59(27.29)$ \\
\hline & $65.70(29.80)^{*}$ & $73.30(27.00)^{*}$ & $63.10(25.10)^{*}$ \\
\hline
\end{tabular}

* Reference population values of Spanish people aged 70 and over [50]. SPMSQ Short Portable Mental Status Questionnaire.

$A D L$ Activities of Daily Living.

$S P P B$ Short Physical Performance Battery.

PF-10 Subscale of physical functioning of the Medical Outcomes Study Short Form-36. 


\section{BP01: Get into bed}
A1
B1
C1
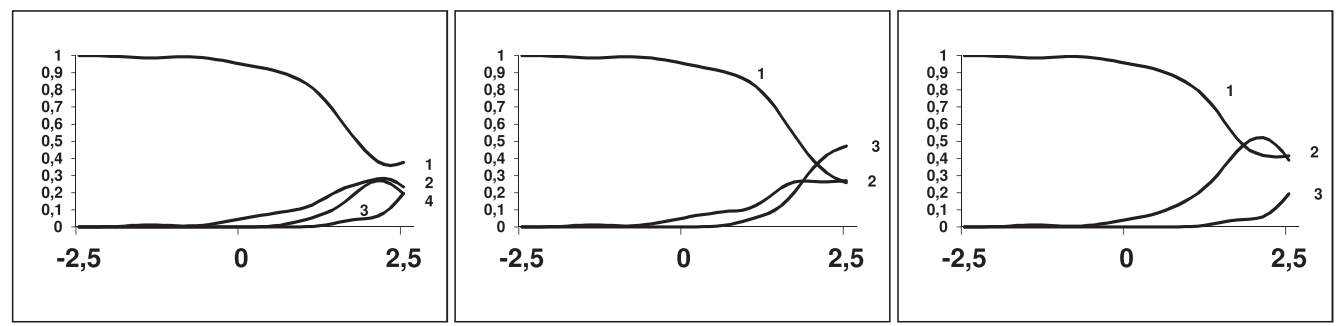

\section{W05: Walk 5 minutes nonstop}

A2

B2

$\mathrm{C} 2$
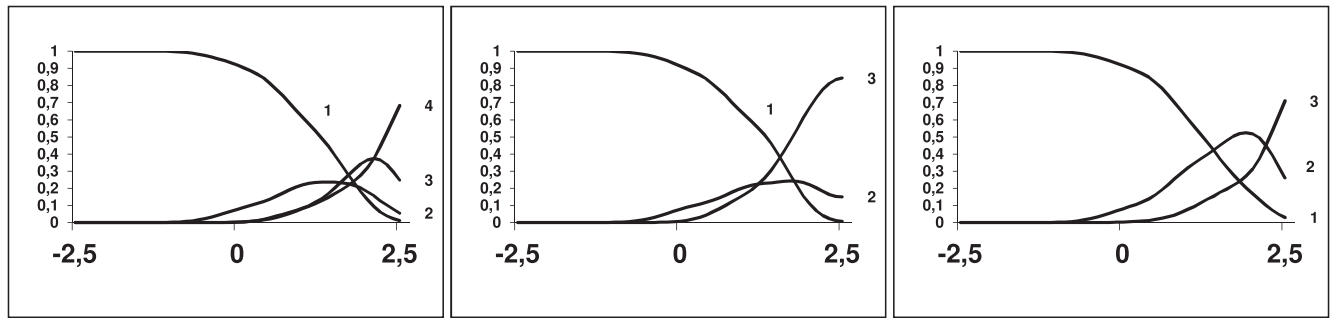

\section{W07: Walk 1 kilometre with rests}

A3

B3

C3
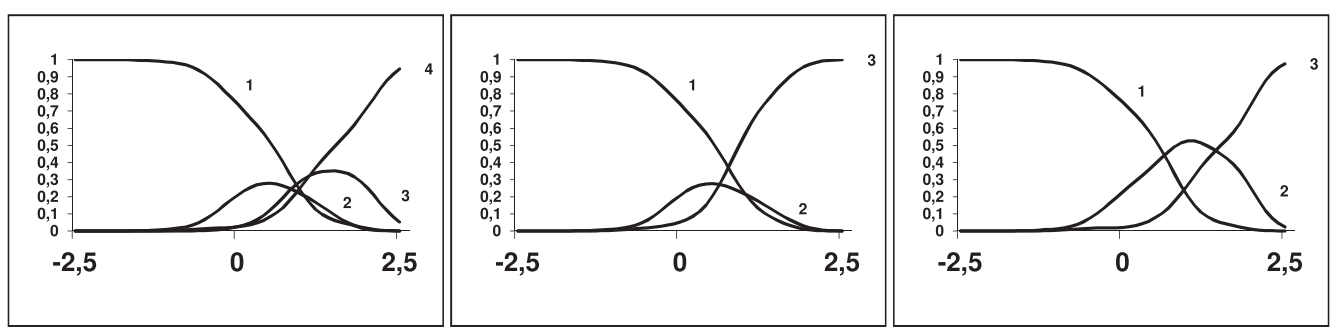

4. W12: Walk several kilometres on uneven ground

A4

B4

C4
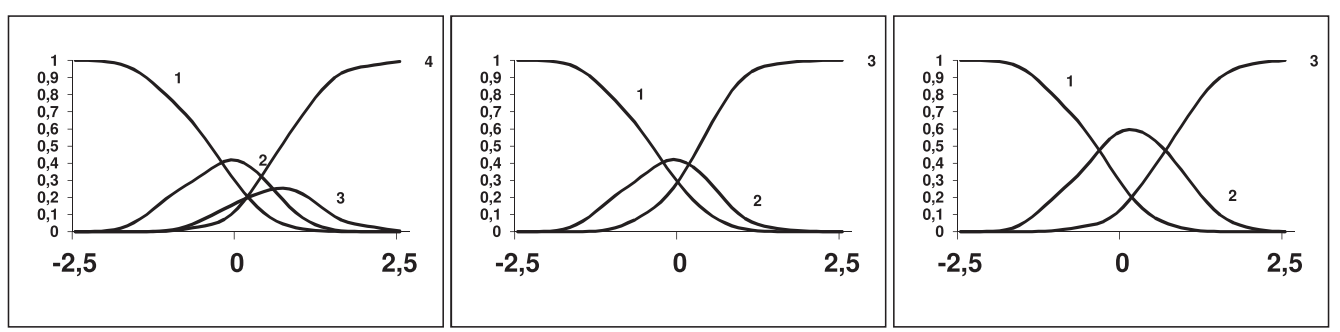

Figure 1 Four examples of different characteristic option curves. Characteristic option curves sorted by degree of difficulty (top-down: lowest to highest difficulty) and by type of rating scale (from left to right: $\mathrm{A}=$ No difficulty; Some difficulty; Much difficulty; Unable to do. B= No difficulty; Some difficulty; Much difficulty + Unable to do. C= No difficulty; Some difficulty + Much difficulty; Unable to do). The first item was very easy and was unsuitable for the sample, although with rating scale type $\mathrm{C}$ it showed good behaviour. The second and third items only presented the desired features with rating scale type $C$, while the fourth one showed the desired features with rating scale types B and $C$. 
[34]. The RSM enables estimates of item location, category thresholds and subject score to be placed on the same metric. The fit of data to the RSM was assessed with infit and outfit mean square error statistics, using a cut-off of $<0.6$ or $>1.4$ for possible item deletion $[9,44]$. Item deletion was implemented sequentially and concluded once none of the remaining items showed misfit. To assess the accuracy of the final item bank, the test information function and its reciprocal [46] the standard error function, were calculated. The person reliability index (analogous to Cronbach's alpha, but excluding extreme scores [47]) was also calculated. To examine item bank coverage and suitability for the sample, item difficulties and person scores were plotted together, centering the scale on zero logitsthe average difficulty of items. Finally, the mobility item bank and the PF-10 items were grouped according to their response options and then co-calibrated onto one common construct (mobility). We used the same pivot anchor for both rating scales: the step from "no difficulty" (or "no limitation") to the next [48]. WINSTEPS was used for these analyses [49].

\section{Missing data}

All of the analyses except the RSM analysis were performed using imputed data obtained through matching, employing the PRELIS (LISREL) Impute Missing Value dialog box. For the RSM analysis, Joint Maximum Likelihood was implemented as the estimation method. This method does not require missing data to be imputed but considers such data ignorable.

\section{Results}

Table 1 presents the demographic and clinical characteristics of the subjects.

\section{Descriptive analysis of the item pool}

Three items returned percentages for the first response option ("no difficulty") of $90 \%$ or more, the item-test correlations ranged between 0.53 and 0.83 and percentages of missing responses per item were less than $5 \%$ in all cases with the exception of two which were slightly higher.

\section{IRT asumptions}

The item response curves had a monotonic relation with the construct for all the items; however, the slopes of three items were not steep enough (items previously identified with percentages $>90 \%$ in the first response option). As regards the characteristic response curves, for the majority of the items the intermediate option curves ("some difficulty", "much difficulty") lacked a maximum over a unique interval of the scale. Therefore, we examined two possibilities: combining both intermediate options or combining the last two options, i.e., no difficulty, some + much difficulty and unable to do, vs. no difficulty, some difficulty, much difficulty + unable to do. The first solution was clearly better since the curves for all the items would then have a maximum over a unique interval of the scale, whilst in the second solution, the curves for the intermediate option would lack a maximum for the majority of the items. Figure 1 shows examples of these curves for four items with each of the three rating scales. Consequently, we eliminated the three items which were flagged and recoded the rating scale for the successive analyses into three categories: no difficulty, some/much difficulty and unable to do.

Table 2 gives the confirmatory factor analysis results both for the entire sample and separately for men and women. Item loadings and fit indices of the single factor model supported a unidimensional interpretation of the item pool. Furthermore, the results for the bifactor model indicated that the influence of the domains (group factors) did not distort this interpretation: the differences between common factor loadings in the bifactor model and the single-factor model did not exceed 0.10 , with a median of 0.01 ; the group factors

Table 2 Model fit statistics for confirmatory factor analyses

\begin{tabular}{|c|c|c|c|c|c|c|}
\hline Models & CFI & NNFI (TLI) & RMSEA & SRMR & Satorra-Bentler $x^{2}$ & df \\
\hline Single & .99 & .99 & .072 & .056 & $3816.72^{*}$ & 945 \\
\hline Bifactor & 1.00 & .99 & .048 & .040 & $2126.52^{*}$ & 900 \\
\hline Single (female) & .99 & .99 & .072 & .061 & $2475.04^{*}$ & 902 \\
\hline Bifactor (female) & .99 & .99 & .051 & .047 & $1599.65^{*}$ & 858 \\
\hline Single (male) & .99 & .99 & .072 & .083 & $2079.19^{*}$ & 902 \\
\hline Bifactor (male) & .99 & .99 & .055 & .067 & $1506.36^{*}$ & 858 \\
\hline Standard cutoff val. & $>.95$ & $>.95$ & $<.06$ & $<.08$ & & \\
\hline
\end{tabular}

CFI Comparative Fit Index.

NNFI (TLI) Non Normed Fit Index (Tucker Lewis Index).

RMSEA Root Mean Square Error of Approximation.

SRMR Standardized Root Mean Square Residual.

$d f$ degree of freedom.

${ }^{*} \mathrm{p}<0.01$. 
Table 3 Differential item functioning (DIF) and differential bundle functioning (DBF) results

\begin{tabular}{|c|c|c|c|c|c|c|c|}
\hline \multirow[t]{3}{*}{ 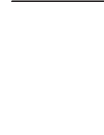 } & \multirow[t]{3}{*}{ Item Wording } & \multirow{2}{*}{\multicolumn{2}{|c|}{ Internal criterion }} & \multicolumn{4}{|c|}{ External criteria } \\
\hline & & & & \multicolumn{2}{|c|}{ PF-10 } & \multicolumn{2}{|c|}{ SPPB } \\
\hline & & Beta & $\mathrm{p}$ & Beta & $\bar{p}$ & Beta & $\mathrm{p}$ \\
\hline \multicolumn{8}{|c|}{ Walking $(\mathbf{W})$} \\
\hline W01 & Walk around the house & -.01 & .75 & -.02 & .41 & -.03 & .32 \\
\hline W02 & Walk without losing balance & .02 & .56 & .03 & .38 & .01 & .71 \\
\hline W03 & Walk without tripping & .05 & .13 & .07 & .03 & .07 & .07 \\
\hline W04 & Walk outdoors & -.06 & .06 & -.05 & .25 & -.08 & .07 \\
\hline W05 & Walk 5 minutes nonstop & -.04 & .23 & -.03 & .40 & -.05 & .19 \\
\hline W06 & Cross the road & .04 & .06 & .05 & .11 & .01 & .82 \\
\hline W07 & Walk 1 kilometre with rests & -.08 & .07 & -.04 & .43 & -.11 & .03 \\
\hline W08 & Walk on a slippery surface & .01 & .80 & .06 & .19 & .01 & .87 \\
\hline W09 & Walk 15 minutes nonstop & -.07 & .08 & -.09 & .04 & -.12 & .02 \\
\hline W10 & Walk 5 minutes at a fast pace & .14 & .01 & -.11 & .05 & -.14 & .01 \\
\hline W11 & Walk 2 kilometres nonstop & -.21 & .00 & -.14 & .01 & -.17 & .00 \\
\hline W12 & Walk several kilometres on uneven ground & -.03 & .58 & .04 & .40 & -.01 & .93 \\
\hline W13 & Walk half an hour at a fast pace nonstop & -.14 & .01 & -.04 & .50 & -.07 & .30 \\
\hline W14 & Climb a steep hill & -.02 & .59 & .07 & .07 & .09 & .06 \\
\hline W15 & Run a short distance & -.05 & .05 & .02 & .68 & -.01 & .91 \\
\hline \multirow[t]{3}{*}{ W16 } & Run one and a half kilometres & -.08 & .06 & .04 & .43 & .02 & .71 \\
\hline & DBF & & & -.14 & & -.58 & \\
\hline & Average DBF (DBF/n items) & & & -.01 & & -.04 & \\
\hline \multicolumn{8}{|c|}{ Up and down stairs (UDS) } \\
\hline UDS01 & Climb 4 or 5 steps, using handrails & .05 & .09 & .03 & .28 & .05 & .19 \\
\hline UDS02 & Step up and down from a curb & .01 & .76 & .06 & .09 & .02 & .58 \\
\hline UDS03 & Climb 4 or 5 steps, without handrails & .06 & .18 & .10 & .05 & .06 & .30 \\
\hline UDS04 & Go down 4 or 5 steps, using handrails & .05 & .11 & .04 & .17 & .03 & .34 \\
\hline UDS05 & Get on and off a bus & .05 & .17 & .11 & .01 & .06 & .14 \\
\hline UDS06 & Go down 4 or 5 steps, without handrails & .06 & .15 & .13 & .01 & .10 & .07 \\
\hline UDS07 & Go up 1 flight of stairs, without handrails & .02 & .74 & .10 & .06 & .08 & .19 \\
\hline UDS08 & Go down 1 flight of stairs, using handrails & .05 & .11 & .06 & .07 & .06 & .10 \\
\hline UDS09 & Climb stairs carrying little weight & -.05 & .17 & -.04 & .29 & -.02 & .61 \\
\hline UDS10 & Go up 3 flights of stairs, using handrails & .02 & .60 & .06 & .13 & .07 & .14 \\
\hline \multirow[t]{3}{*}{ UDS11 } & Go down 3 flights of stairs, using handrails & .01 & .70 & .03 & .42 & .05 & .32 \\
\hline & DBF & & & .68 & & .56 & \\
\hline & Average DBF (DBF/n items) & & & .06 & & .05 & \\
\hline \multicolumn{8}{|c|}{ Body Position (BP) } \\
\hline BP01 & Get into bedt & - & - & - & - & - & - \\
\hline BP02 & Turn over in bed & .01 & .91 & .05 & .29 & .06 & .15 \\
\hline BP03 & Sit down on a couch & .02 & .50 & .05 & .16 & .02 & .54 \\
\hline BP04 & Sitting, bend over to pick something up & -.04 & .32 & -.02 & .66 & .01 & .81 \\
\hline BP05 & Remain seated for 10 minutes without back rest & .07 & .05 & .13 & .00 & .16 & .00 \\
\hline BP06 & Sit up in bed, being lied down & -.03 & .47 & -.01 & .74 & -.00 & .97 \\
\hline BP07 & Sit down and stand up from a chair $\dagger$ & - & - & - & - & - & - \\
\hline BP08 & Stand up from a low, soft couch & .10 & .03 & .09 & .04 & .08 & .11 \\
\hline
\end{tabular}


Table 3 Differential item functioning (DIF) and differential bundle functioning (DBF) results (Continued)

\begin{tabular}{|c|c|c|c|c|c|c|c|}
\hline BP09 & Pick up a chairt & - & - & - & - & - & - \\
\hline BP10 & Reach overhead while standing & .19 & .00 & .22 & .00 & .26 & .00 \\
\hline BP11 & Turn around while standing & .07 & .09 & .08 & .04 & .09 & .04 \\
\hline BP12 & Remain standing for 10 minutes & -.03 & .50 & -.01 & .83 & .00 & .98 \\
\hline BP13 & Standing, bend down to pick something up & -.04 & .37 & .01 & .80 & .01 & .80 \\
\hline BP14 & Get up from the floor from lying on your back & .20 & .00 & .24 & .00 & .23 & .00 \\
\hline BP15 & Kneel down & .29 & .00 & .32 & .00 & .32 & .00 \\
\hline \multirow[t]{3}{*}{ BP16 } & Get into and out of a car & .03 & .53 & .08 & .06 & .08 & .06 \\
\hline & DBF & & & 1.23 & & 1.32 & \\
\hline & Average DBF (DBF/n items) & & & .09 & & .10 & \\
\hline \multicolumn{8}{|c|}{ Carrying, Lifting and Pushing (CLP) } \\
\hline CLP01 & Push or pull a large object & .07 & .09 & .12 & .01 & .13 & .01 \\
\hline CLP02 & Move or drag a bed & .11 & .02 & .13 & .00 & .16 & .00 \\
\hline CLP03 & Lift 4 or $5 \mathrm{~kg}$ from the floor & .17 & .00 & .22 & .00 & .25 & .00 \\
\hline CLP04 & Turn over a mattress & .10 & .04 & .14 & .00 & .18 & .00 \\
\hline \multirow[t]{3}{*}{ CLP05 } & Change gas bottle & .23 & .00 & .22 & .00 & .23 & .00 \\
\hline & DBF & & & .83 & & .95 & \\
\hline & Average DBF (DBF/n items) & & & .17 & & .19 & \\
\hline
\end{tabular}

DIF: in bold, significant values $p<0.002 ;$ DBF: in bold, significant values $p<0.01$.

† Items excluded after Testgraf analysis.

explained only $9.29 \%$ of variance vs. $66.43 \%$ for the common factor, and no item had a higher loading for the group factor than for the common factor. This pattern of results was repeated in analyses by sex, although the influence of the CLP group factor was higher in men.

All the residual correlations for the single-factor model were lower than 0.2 , except one which was 0.21 (the items "Sitting, bend over to pick something up" and "Standing, bend down to pick something up"); consequently, we considered that there were no local dependencies in the item pool.

\section{DBF and DIF analysis}

Standard DIF analysis with the purified conditioning variable flagged the same items with significant DIF as the DIF analysis with no purified conditioning variable. Table 3 gives a summary of DIF results. No item was flagged for non-uniform DIF, but there was evidence of uniform DIF: one item from the Walking domain (W11), three from the BP domain (BP10, BP14, BP15) and two from the CLP domain (CLP03, CLP05) were flagged for significant DIF. No item from the UDS domain was flagged for significant DIF. Furthermore, most of the Walking domain items presented negative (nonsignificant) DIF and all the CLP domain items showed positive (significant or nonsignificant) DIF.

DIF analysis with the two external criteria as conditioning variables produced very similar results: most of the CLP domain items showed significant item DIF, and the BP domain items which were flagged for significant item DIF were the same as those which had been flagged by the standard item DIF analysis. The results of DBF analysis also coincided with the two external criteria: three domains presented DBF (the Walking domain was the exception), but the magnitude was only substantial and consistent across the items in the CLP domain (Table 3).

We have decided to delete items that were consistently (by the three criteria) flagged for significant DIF, but we kept one of them (BP14) because it measured in the highest level of the construct.

\section{Rasch RSM analysis}

Six items, one from the Walking, one from the UDS and four from the BP domains were iteratively eliminated because of misfit. Table 4 shows the category thresholds, item locations and mean square error statistics for the remaining 35 items (15 Walking items, 10 UDS items, 7 $\mathrm{BP}$ items and 3 CLP items). Item pool coverage and accuracy was satisfactory throughout the entire continuum of mobility, with the exception of the upper level of capacity, which corresponds to more demanding activities than running $500 \mathrm{~m}$ without difficulty or performing vigorous activities (Figures 2 and 3). 6.7\% of people obtained the lowest score (greatest capacity or least mobility limitation) and no person received the maximum score. The person reliability index was 0.95 . Figures 2 and 3 also show the results for co-calibration of LLM-IB and PF-10. 
Table 4 Summary of Rasch rating scale analysis and confirmatory factor analysis (loadings)

\begin{tabular}{|c|c|c|c|c|c|c|c|c|c|c|}
\hline & & \multicolumn{6}{|c|}{ Confirmatory factor analysis } & \multicolumn{3}{|c|}{ Rasch rating scale analysis } \\
\hline & & \multirow{2}{*}{$\begin{array}{l}\text { Single } \\
\text { Factor }\end{array}$} & \multicolumn{5}{|c|}{ Bi-factor model } & \multirow{2}{*}{$\begin{array}{l}\text { Location } \\
\text { (Error) }\end{array}$} & \multirow{2}{*}{$\begin{array}{c}\text { Infit } \\
\text { MNSQ }\end{array}$} & \multirow{2}{*}{$\begin{array}{l}\text { Outfit } \\
\text { MNSO }\end{array}$} \\
\hline & & & $\mathbf{G}$ & g1 & g2 & g3 & 94 & & & \\
\hline \multicolumn{11}{|c|}{ Walking $(\mathrm{W})$} \\
\hline W01 & Walk around the house & .88 & .86 & .27 & & & & $3.15(.15)$ & .88 & .54 \\
\hline W02 & Walk without losing balance** & .81 & .80 & .16 & & & & - & - & - \\
\hline W03 & Walk without tripping & .78 & .78 & .06 & & & & $1.71(.11)$ & 1.02 & 1.47 \\
\hline W04 & Walk outdoors & .89 & .86 & .29 & & & & $.91(.10)$ & .78 & .72 \\
\hline W05 & Walk 5 minutes nonstop & .83 & .79 & .33 & & & & $2.12(.12)$ & 1.13 & .73 \\
\hline W06 & Cross the road & .87 & .87 & .17 & & & & $2.02(.12)$ & .91 & .77 \\
\hline W07 & Walk 1 kilometre with rests & .89 & .83 & .40 & & & & $.50(.09)$ & .98 & .81 \\
\hline W08 & Walk on a slippery surface & .82 & .82 & .14 & & & & $-.84(.09)$ & .95 & 1.29 \\
\hline W09 & Walk 15 minutes nonstop & .88 & .81 & .46 & & & & $.84(.10)$ & 1.03 & .95 \\
\hline W10 & Walk 5 minutes at a fast pace & .87 & .80 & .44 & & & & $-.74(.09)$ & 1.22 & 1.00 \\
\hline W11 & Walk 2 kilometres nonstop & .90 & .84 & .43 & & & & $-.66(.09)$ & 1.03 & .83 \\
\hline W12 & Walk several kilometres on uneven ground & .88 & .85 & .27 & & & & $-1.53(.08)$ & .90 & .84 \\
\hline W13 & Walk half an hour at a fast pace nonstop & .88 & .81 & .46 & & & & $-2.47(.09)$ & 1.04 & .90 \\
\hline W14 & Climb a steep hill & .81 & .80 & .20 & & & & $-1.06(.09)$ & .89 & 1.29 \\
\hline W15 & Run a short distance & .88 & .86 & .26 & & & & $-2.31(.09)$ & 1.08 & .89 \\
\hline W16 & Run one and a half kilometres & .83 & .80 & .30 & & & & $-4.30(.10)$ & 1.29 & .97 \\
\hline \multicolumn{11}{|c|}{ Up and down stairs (UDS) } \\
\hline UDS01 & Climb 4 or 5 steps, using handrails & .86 & .83 & & .40 & & & $1.89(.11)$ & .87 & .72 \\
\hline UDSO2 & Step up and down from a curb & .86 & .87 & & .11 & & & $1.42(.11)$ & .81 & .80 \\
\hline UDS03 & Climb 4 or 5 steps, without handrails & .93 & .93 & & .16 & & & $-.31(.09)$ & .97 & .77 \\
\hline UDSO4 & Go down 4 or 5 steps, using handrails** & .80 & .75 & & .54 & & & - & - & - \\
\hline UDS05 & Get on and off a bus & .88 & .89 & & .03 & & & $.22(.09)$ & .74 & .69 \\
\hline UDS06 & Go down 4 or 5 steps, without handrails & .92 & .92 & & .15 & & & $-.47(.09)$ & .98 & .87 \\
\hline UDS07 & Go up 1 flight of stairs, without handrails & .90 & .91 & & .10 & & & $-1.54(.08)$ & .91 & .88 \\
\hline UDS08 & Go down 1 flight of stairs, using handrails & .90 & .88 & & .34 & & & $1.42(.11)$ & .74 & .70 \\
\hline UDS09 & Climb stairs carrying little weight & .87 & .86 & & .26 & & & $.64(.10)$ & .87 & .83 \\
\hline UDS10 & Go up 3 flights of stairs, using handrails & .88 & .88 & & .22 & & & $-.98(.09)$ & .75 & .79 \\
\hline UDS11 & Go down 3 flights of stairs, using handrails & .89 & .88 & & .26 & & & $-.29(.09)$ & .80 & .75 \\
\hline \multicolumn{11}{|c|}{ Body positions (BP) } \\
\hline BP01 & Get into bed $t$ & - & - & & & & & - & - & - \\
\hline BP02 & Turn over in bed** & .62 & .60 & & & .32 & & - & - & - \\
\hline $\mathrm{BPO3}$ & Sit down on a couch & .83 & .83 & & & .23 & & $1.48(.11)$ & 1.18 & .80 \\
\hline BP04 & Sitting, bend over to pick something up & .75 & .72 & & & .46 & & $0.83(.10)$ & 1.25 & 1.23 \\
\hline BP05 & Remain seated for 10 minutes without back rest** & .76 & .76 & & & .20 & & - & - & - \\
\hline BP06 & Sit up in bed, being lied down** & .72 & .71 & & & .25 & & - & - & - \\
\hline BP07 & Sit down and stand up from a chairt & - & - & & & & & - & - & - \\
\hline BP08 & Stand up from a low, soft couch & .80 & .79 & & & .21 & & $-.87(.09)$ & 1.01 & 1.16 \\
\hline BP09 & Pick up a chairt & - & - & & & & & - & - & - \\
\hline BP10 & Reach overhead while standing* & .74 & .73 & & & .26 & & - & - & - \\
\hline BP11 & Turn around while standing ${ }^{* *}$ & .70 & .69 & & & .23 & & - & - & - \\
\hline BP12 & Remain standing for 10 minutes & .82 & .83 & & & .03 & & $-.15(.09)$ & 1.14 & 1.06 \\
\hline
\end{tabular}


Table 4 Summary of Rasch rating scale analysis and confirmatory factor analysis (loadings) (Continued)

\begin{tabular}{|c|c|c|c|c|c|c|c|c|}
\hline BP13 & Standing, bend down to pick something up & .79 & .77 & .47 & & $.02(.09)$ & 1.12 & 1.03 \\
\hline BP14 & Get up from the floor from lying on your back & .83 & .82 & .29 & & $-1.96(.08)$ & .95 & 1.03 \\
\hline BP15 & Kneel down* & .79 & .79 & .19 & & - & - & - \\
\hline BP16 & Get into and out of a car & .76 & .76 & .20 & & $.54(.09)$ & 1.10 & 1.31 \\
\hline \multicolumn{9}{|c|}{ Carrying, lifting and pushing (CLP) } \\
\hline CLP01 & Push or pull a large object & .80 & .79 & & .42 & $.26(.09)$ & 1.20 & 1.20 \\
\hline CLP02 & Move or drag a bed & .81 & .79 & & .48 & $.12(.09)$ & 1.21 & 1.33 \\
\hline CLP03 & Lift 4 or $5 \mathrm{~kg}$ from the floor* & .80 & .80 & & .30 & - & - & - \\
\hline CLP04 & Turn over a mattress & .81 & .80 & & .34 & $.36(.09)$ & 1.21 & 1.14 \\
\hline CLP05 & Change gas bottle* & .82 & .81 & & .39 & - & - & - \\
\hline
\end{tabular}

RSM category thresholds: -1.34 (no difficulty vs. difficulty), 1.34 (difficulty vs. unable to do).

† Items excluded after Testgraf analysis.

* Items excluded after DIF analysis.

** Items excluded (misfit) after Rasch rating scale analysis.

$\mathrm{G}$ - General factor.

g- Group factor.

MNSQ Mean square error.

\section{Discussion}

In this paper, we present the development and validation of a mobility item pool in a sample of 593 older people attending primary health care practices in Spain. Item content was based on ICF mobility indicators, and the item stems and response options concerned difficulty in performing an activity without external help. We examined IRT assumptions, analysed DIF/DBF by sex and calibrated the item pool with the Rasch RSM. No evidence was found of non-uniform DIF but we did observe uniform DIF and DBF. Although the confirmatory factor analysis results satisfied stringent criteria for unidimensionality, the DBF results called this conclusion into question, mainly because with the exception of the Walking domain, all other domains showed DBF, notably the CLP domain. Following the Rasch RSM analysis, 35 items remained in the pool and formed the Late Life Mobility item bank (LLM-IB), which measured with a reliability of 0.90 or higher across the entire spectrum of mobility, except at the extreme end of better function. Lastly, the 35 items were co-calibrated with the PF-10 items.

A noteworthy aspect of this study is that to the best of our knowledge, this is the first time in the literature on patient reported outcomes that DBF has been analysed. To achieve this, in addition to examining DIF according to

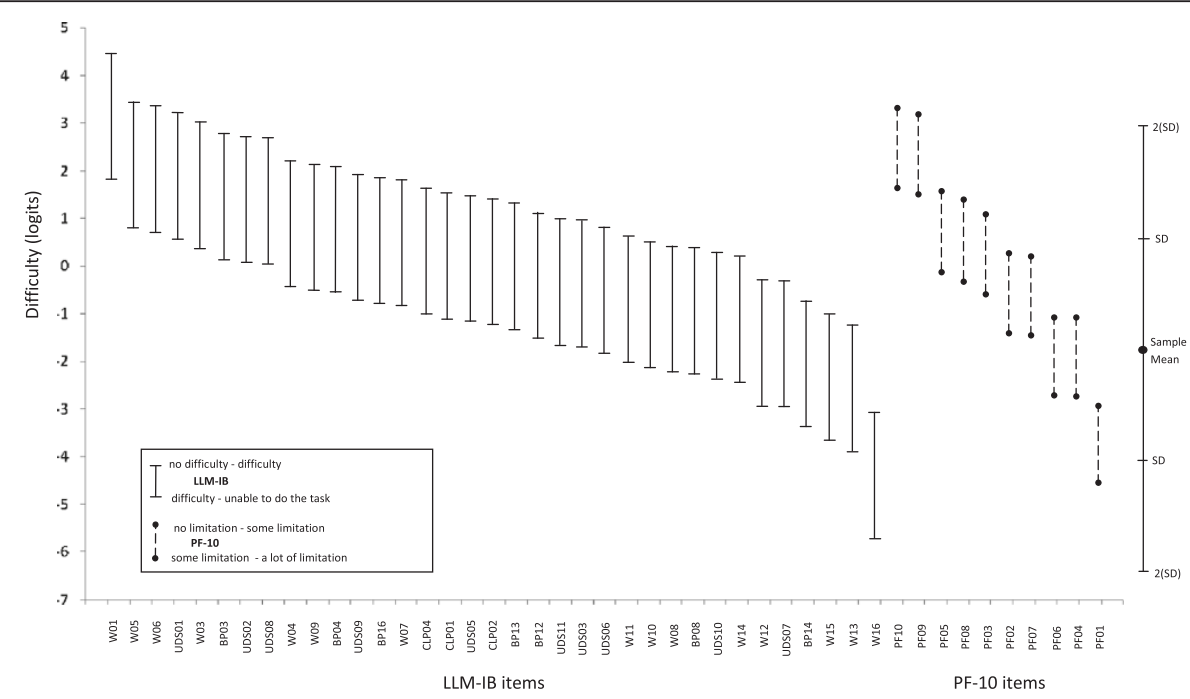

Figure 2 Coverage and suitability of the item bank for the sample. Difficulties of the items and scores of subjects are plotted together. On the left: items from the Late Life Mobility item bank (LLM-IB), on the right: items from the PF-10 subscale. W: items from the walking domain, UDS: items from the going up and down stairs domain, BP: items from the changing and maintaining body position domain, CLP: items from the carrying, lifting and pushing domain and PF: items from the PF-10 subscale. 


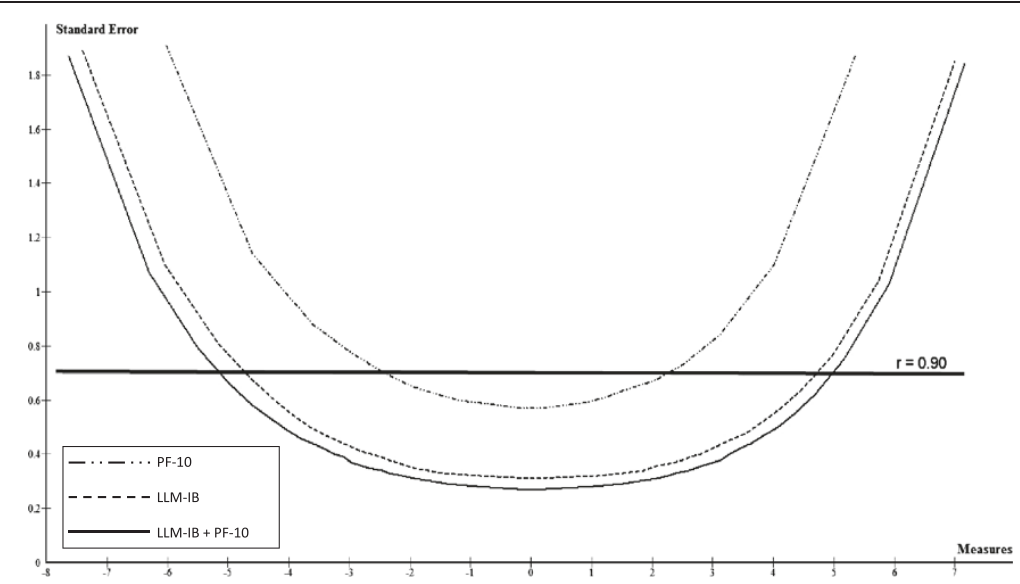

Figure 3 Standard error functions of the Late Life Mobility item bank (LLM-IB), PF-10 subscale and Late Life Mobility item bank + PF-10 subscale.

standard procedure, we also examined augmented DIF at domain level (DBF) using two external criteria as conditioning variables: the PF-10 scale and SPPB. Results of the DIF/ DBF analysis with the two external criteria were very similar, suggesting no bias in self-report versus performancebased scales as a method to measure late life mobility: most of the CLP domain items and three BP domain items were flagged for significant DIF. Standard DIF results were less similar to those above, since fewer CLP domain items were identified as presenting significant DIF and there were more items with DIF, significant or nonsignificant, with opposite signs: most of the Walking domain items were negative and all of the CLP domain items were positive. This has also been observed recently during the development of the European Organisation for Research and Treatment of Cancer Physical Function item bank, and the most plausible explanation is that both bundles/domains measure different secondary dimensions $[24,25,35]$.

Although conditioning with an internal criterion such as total score produces DIF values with a trade off between positive and negative values as DIF values are statistically dependent [26], it is interesting that the items which systematically presented opposite values were Walking and CLP items. However, when an external criterion is used as a conditioning variable, statistical dependence disappears [26]. Thus, DIF/DBF analysis using SPPB and PF-10 as conditional variables revealed that CLP measured a secondary dimension that produced significant DIF and DBF, but Walking domain items produced neither DBF nor DIF, with the exception of one item according to SPPB but none according to PF-10. Therefore, standard DIF analysis indicated that Walking items and CLP items measured different domains and DIF/DBF analysis revealed that Walking was the core dimension of the mobility construct and CLP was a secondary dimension that produced DBF. This interpretation, that CLP items measure a secondary dimension of the mobility construct, is also consistent with results from non-psychometric studies, which have reported that gender differences are greater in items in this domain than in other mobility domains [16-21] and that these differences do not disappear after adjustment for important covariables $[18,19,51]$. These results are also consistent with those found in the fields of geriatric frailty and sarcopenia, where these items are commonly referred to as indicators of strength: walking and strength constitute two separate sub-dimensions of the frailty construct [52,53], and strength is a predictor of mobility decline and is a more intense predictor in men than women [54]. If a secondary dimension produces DIF, the DIF is benign if the dimension is considered part of the construct, but adverse if the secondary dimension is considered a nuisance $[25,40]$. Therefore, deciding whether the strength domain produces benign DIF or adverse DIF is a theoretical issue, but the data show that the inclusion of strength items increases gender differences in mobility. When validating the LLMIB, we decided that the strength domain produces benign DBF and we excluded only those items that were consistently flagged for significant DIF.

We used the Rasch RSM to calibrate the item bank and eliminated six of the 41 items that still remained in the item pool, having previously eliminated three for being too easy and four due to DIF. Thus, 35 items remained and constituted the LLM-IB. Most of the Walking and UDS items were retained since they did not present any of the problems observed in the items in the other two domains. We believe that these results help to explain the predominance of walking and going up \& down stairs items in the fixed and adaptive physical function measures. Indeed, in the PF-10 and Health Assessment Questionnaire II [55], most of the items are from the Walking or UDS domains. In the new measures, short forms and computer adaptive test applications developed from item 
banks such as the Patient Reported Outcomes Measurement Information System Physical Function item bank [56] or the Activity Measure for Post Acute Care mobility item bank [6] also produce a predominance of items from the Walking and UDS domains. This occurs even if a content balancing algorithm is introduced to select the first items from the computer adaptive test applications, since the greater wealth of information contained in the Walking and UDS items, calibrated with IRT models which included a discrimination parameter, means that in the end, these achieve greater representation.

The item pool originally contained four response options, but a graphical, non-parametric IRT analysis showed that the number of response options per item should be reduced. We examined two rating scale alternatives, one combining the two intermediate options ("some difficulty" and "much difficulty") whilst the other combined the two options reflecting greatest difficulty ("much difficulty" and "unable to do"). We chose the first because it was psychometrically better, and because it is common practice to distinguish between difficulty and incapacity in research on the disablement process. Our sample consisted of older people, generally with a poor educational level (reflecting the current cohort of the elderly population in Spain), which alone may explain why a rating scale with three options works better than a rating scale with more [57].

This study has various limitations. Firstly, in the DBF analysis, one of the bundles, CLP domain, contained only five items. Consequently, the idiosyncrasy of these may constitute an alternative explanation to our interpretation based on the validity of five items as a domain measure. However, the items included are among the most common in the literature. In addition, care was taken not to include items that were too demanding and which would thus have favoured men even more. Secondly, although the use of two conditioning variables which are widely accepted as standard physical function and mobility measures is one of the strengths of this analysis, the study lacked a similar standard for the CLP domain: an objective measure of strength would have enhanced the construct validity of the findings. Thirdly, because DIF by age has repeatedly been found for many items in measures of PF, the extrapolation of our results beyond samples of older people is questionable. Finally, our findings are exclusively cross-sectional. We anticipate validating the item bank and several fixed forms with the longitudinal data collected after monitoring the same cohort for 18 months with outcome variables such as mortality, dependency and hospitalization.

\section{Conclusions}

We have designed an item bank in Spanish to measure mobility in older primary care patients which is free from item bias across gender and was calibrated using Rasch RSM. Item bank accuracy and coverage was satisfactory throughout the entire continuum of mobility, with the exception of the upper level of capacity, suggesting the desirability of replenishing the item bank with items that measure at high mobility function level. Furthermore, our results indicate that the walking and going up and down stairs items form the core of the mobility construct whilst strength items form a secondary dimension that produces augmented DIF. These results highlight the desirability of stratifying by domain and weighting domain representation when selecting items to create fixed or adaptive forms of mobility for older people, leaving only strength items marginal. Further research is needed to determine how best to include strength items in a mobility measure, or whether it would be more appropriate to design separate measures for each construct.

\section{Abbreviations}

PF-10: Physical Functioning subscale of the Medical Outcomes Study Short Form-36; IRT: Item Response Theory; ICF: International Classification of Functioning, Disability and Health; DIF: Differential item functioning; DBF: Differential bundle functioning; LLM-IB: Late Life Mobility Item Bank; BP: Changing and maintaining body position mobility domain; CLP: Carrying, lifting and pushing mobility domain; UDS: Going up and down stairs mobility domain; SPPB: Short Physical Performance Battery; ADL: Activity of daily living; RSM: Rating scale model; CFI: Comparative fit index; TLI: Tucker Lewis index; RSMEA: Root-mean-square error of approximation;

SRMR: Standardised root mean square residual; SIBTEST: Simultaneous item bias test.

\section{Competing interests}

The authors declare that they have no competing interests.

\section{Authors' contributions}

JCG conceived the study, performed statistical analysis and drafted the manuscript. JDRP, CLMM, MJCM, LG participated in data collection and management. All authors participated in the design of the study and interpretation of data. All authors read and approved the final manuscript.

\section{Acknowledgements}

This research has been supported by a grant from the Spanish Ministry of Health and Social Policy (PI05/1538, Health Research Fund). We would particularly like to thank the contribution of Professor Miguel RichartMartínez, PhD as focus groups moderator. We also thank Dr. Nieves GómezMoreno, Dr. Soledad García-Beviá and others physicians and nurses of Valencian Health Agency primary care services who helped us in data collection.

\section{Author details}

${ }^{1}$ Department of Nursing, University of Alicante, Ctra. San Vicente $s / n$, San Vicente del Raspeig, Alicante 03015, Spain. ${ }^{2}$ Department of Health, Alcoy, Alicante, Spain. ${ }^{3}$ Department of Health Psychology, University of Alicante, Alicante, Spain.

\section{Received: 19 August 2012 Accepted: 29 November 2012}

Published: 5 December 2012

\section{References}

1. Smith KW, Avis NE, Assmann SF: Distinguishing between quality of life and health status in quality of life research: a meta-analysis. Qual Life Res 1999, 8:447-459.

2. Bruce B, Fries JF: The Stanford Health Assessment Questionnaire: dimensions and practical applications HAQ. Health Qual Life Outcomes 2003, 1:20. 
3. Haley SM, McHorney CA, Ware JE Jr: Evaluation of the MOS SF-36 physical functioning scale (PF-10): I. Unidimensionality and reproducibility of the Rasch item scale. J Clin Epidemiol 1994, 47:671-684.

4. Reeve BB, Hays RD, Bjorner JB, Cook KF, Crane PK, Teresi JA, Revicki DA, Weiss DJ, Hambleton RK, Liu H, et al: Psychometric evaluation and calibration of health-related quality of life item banks: plans for the Patient-Reported Outcomes Measurement Information System (PROMIS). Med Care 2007, 45(Suppl 1):S22-S31.

5. Fries JF, Bruce B, Bjorner JB, Rose M: More relevant, precise, and efficient items for assessment of physical function and disability: moving beyond the classic instruments. Ann Rheum Dis 2006, 65(Suppl 3):iii16-iii21.

6. Jette AM, Haley SM, Tao W, Ni P, Moed R, Meyers D, Zurek M: Prospective evaluation of the AM-PAC-CAT in outpatient rehabilitation settings. Phys Ther 2007, 87:385-398.

7. DeWitt EM, Stucky BD, Thissen D, Irwin DE, Langer M, Varni JW, Lai JS, Yeatts $K B$, Dewalt DA: Construction of the eight-item patient-reported outcomes measurement information system pediatric physical function scales: built using item response theory. J Clin Epidemiol 2011, 64:794-804.

8. Rose M, Bjorner JB, Becker J, Fries JF, Ware JE: Evaluation of a preliminary physical function item bank supported the expected advantages of the Patient-Reported Outcomes Measurement Information System (PROMIS). J Clin Epidemiol 2008, 61:17-33.

9. Hart DL, Wang YC, Stratford PW, Mioduski JE: Computerized adaptive test for patients with foot or ankle impairments produced valid and responsive measures of function. Qual Life Res 2008, 17:1081-1091.

10. Holman R, Weisscher N, Glas CA, Dijkgraaf MG, Vermeulen M, de Haan RJ, Lindeboom R: The Academic Medical Center Linear Disability Score (ALDS) item bank: item response theory analysis in a mixed patient population. Health Qual Life Outcomes 2005, 3:83.

11. Haley SM, Jette AM, Coster WJ, Kooyoomijan JT, Levenson S, Heeren T, Ashba J: Late Life Function and Disability Instrument: II. Development and evaluation of the function component. J Gerontol A Biol Sci Med Sci 2002, 57:M217-M222.

12. Jette AM, Haley SM, Ni P, Olarsch S, Moed R: Creating a computer adaptive test version of the late-life function and disability instrument. J Gerontol A Biol Sci Med Sci 2008, 63:1246-1256.

13. Guralnik JM, Ferrucci L: Assessing the building blocks of function: utilizing measures of functional limitation. Am J Prev Med 2003, 25(Suppl 2):112-121.

14. Gill TM: Assessment of function and disability in longitudinal studies. J Am Geriatr Soc 2010, 58(Suppl 2):S308-S312.

15. World Health Organization: International Classification of Functioning, Disability and Health. Geneva; 2001.

16. Leveille SG, Penninx BW, Melzer D, Izmirlian G, Guralnik JM: Sex differences in the prevalence of mobility disability in old age: the dynamics of incidence, recovery, and mortality. J Gerontol B Psychol Sci Soc Sci 2000, 55:S41-S50.

17. Freedman VA, Martin LG: Understanding trends in functional limitations among older Americans. Am J Public Health 1998, 88:1457-1462.

18. Murtagh KN, Hubert HB: Gender differences in physical disability among an elderly cohort. Am J Public Health 2004, 94:1406-1411.

19. Wray LA, Blaum CS: Explaining the role of sex on disability: a populationbased study. Gerontologist 2001, 41:499-510.

20. Simonsick EM, Newman AB, Nevitt MC, Kritchevsky SB, Ferrucci L, Guralnik $J M$, Harris T, ABC Health Study Group: Measuring higher level physical function in well-functioning older adults: expanding familiar approaches in the health ABC study. J Gerontol A Biol Sci Med Sci 2001, 56:M644-M649.

21. Kuh D, Bassey EJ, Butterworth S, Hardy R, Wadsworth ME, Musculoskeletal Study Team: Grip strength, postural control, and functional leg power in a representative cohort of British men and women: associations with physical activity, health status, and socioeconomic conditions. J Gerontol A Biol Sci Med Sci 2005, 60:224-231.

22. Sainio $P$, Koskinen $S$, Heliövaara M, Martelin T, Härkänen T, Hurri H, Miilunpalo S, Aromaa A: Self-reported and test-based mobility limitations in a representative sample of Finns aged 30+. Scand J Public Health 2006, 34:378-386

23. Teresi JA, Ocepek-Welikson K, Kleinman M, Cook KF, Crane PK, Gibbons LE, Morales LS, Orlando-Edelen M, Cella D: Evaluating measurement equivalence using the item response theory log-likelihood ratio (IRTLR) method to assess differential item functioning (DIF): applications (with illustrations) to measures of physical functioning ability and general distress. Qual Life Res 2007, 16(Suppl 1):43-68.
24. Petersen MA, Groenvold M, Aaronson NK, Chie WC, Conroy T, Costantini A, Fayers P, Helbostad J, Holzner B, Kaasa S, et al: Development of computerized adaptive testing (CAT) for the EORTC QLQ-C30 physical functioning dimension. Qual Life Res 2011, 20:479-490.

25. Langer MM, Hill CD, Thissen D, Burwinkle TM, Varni JW, Dewalt DA: Item response theory detected differential item functioning between healthy and ill children in quality-of-life measures. J Clin Epidemiol 2008, 61:268-276.

26. Scott NW, Fayers PM, Aaronson NK, Bottomley A, de Graeff A, Groenvold M, Gundy C, Koller M, Petersen MA, Sprangers MA, et al: Differential item functioning (DIF) analyses of health-related quality of life instruments using logistic regression. Health Qual Life Outcomes 2010, 8:81.

27. Roussos L, Stout W: A multidimensionality-based DIF analysis paradigm. Appl Psychol Meas 1996, 20:355-371.

28. Pfeiffer E: A short portable mental status questionnaire for the assessment of organic brain deficit in elderly patients. J Am Geriatr SoC 1975, 23:433-441.

29. Martin M, Kosinski M, Bjorner JB, Ware JE Jr, Maclean R, Li T: Item response theory methods can improve the measurement of physical function by combining the modified health assessment questionnaire and the SF-36 physical function scale. Qual Life Res 2007, 16:647-660

30. Working Group on Functional Outcome Measures for Clinical Trials: Functional outcomes for clinical trials in frail older persons: time to be moving. J Gerontol B Psychol Sci Soc Sci 2008, 63:160-164.

31. Sheik JI, Yesavage JA: Geriatric Depression Scale (GDS). Recent evidence and development of shorter version. Clin Gerontol 1986, 5:173.

32. Fried LP, Guralnik JM: Disability in older adults: evidence regarding significance, etiology, and risk. J Am Geriatr Soc 1997, 45:92-100.

33. Fried LP, Bandeen-Roche K, Chaves PH, Johnson BA: Preclinical mobility disability predicts incident mobility disability in older women. $J$ Gerontol A Biol Sci Med Sci 2000, 55:M43-M52.

34. Andrich D: A rating formulation for ordered response categories. Psychometrika 1978, 43:561-573.

35. Groenvold M, Petersen MA: The role and use of the diferential item functioning (DIF) analysis of quality of life data from clinical trials. In Assessing quality of life in clinical trials. Edited by Fayers P, Hays R. Oxford: Oxford University Press; 2005:195-208

36. Ramsay JO: Testgraf. A program for the graphical analysis of multiple choice test and questionnaire data. [computer software]. Montreal: Department of Psychology, McGill University; 2000.

37. Jöreskog KG, Sörbom D, Jöreskog KG, Sörbom D: LISREL [computer software]. Version 8.80. Chicago: Scientific Software International; 2006.

38. Reise SP, Morizot J, Hays RD: The role of the bifactor model in resolving dimensionality issues in health outcomes measures. Qual Life Res 2007, 16(Suppl 1):31.

39. $\mathrm{Hu}$ L, Bentler PM: Cutoff criteria for fit indexes in covariance structure analysis: conventional criteria versus new alternatives. Struct Equ Modeling 1999, 6:1-55.

40. Roussos LA, Stout W: Differential item functioning analysis: detecting DIF item and testing DIF hipothesis. In The Sage handbook of quantitative methodology for the social sciences. Edited by Kaplan D. Thousand Oaks: Sage Publications, Inc; 2004:107-115.

41. William Stout Institute for Measurement: Dimensionality-based DIF analysis package: SIBTEST, Poly-SIBTEST, Crossing SIBTEST, DIFSIM, DIFCOMP [computer software]. Version 1.2. Champaign, IL: Assessment Corporation; 2005.

42. Chang H, Mazzeo J, Roussos L: Detecting DIF for Polytomously Scored Items: An Adaptation of the SIBTEST Procedure. J Educ Meas 1996, 33:333-353

43. Li H, Stout W: A new procedure for detection of crossing DIF. Psychometrika 2010, 61:647-677.

44. Bond TG, Fox CM: Applying the Rasch Model: fundamental measurement in the Human Sciences. Mahwah, New Jersey: Lawrence Erlbaum Associates; 2007.

45. Lai JS, Cella D, Chang CH, Bode RK, Heinemann AW: Item banking to improve, shorten and computerize self-reported fatigue: an illustration of steps to create a core item bank from the FACIT-Fatigue Scale. Qual Life Res 2003, 12:485-501.

46. Hambleton $\mathrm{R}$, Swaminathn $\mathrm{H}$, Rogers HJ: Fundamentals of Item Response Theory. Newbury Park, California: Sage Publications, Inc.; 1991.

47. Smith EV: Evidence for the reliability of measures and validity of measure interpretation: a Rasch measurement perspective. In Introduction to Rasch measurement. Edited by Smith EV, Smith RM. Maple Grove, MN: JAM Press; 2004:93-122. 
48. Bode RK: Partial credit model and pivot anchoring. In Introduction to Rasch measurement. Edited by Smith EV, Smith RM. Maple Grove, MN: JAM Press; 2004:279-295.

49. Linacre JM: Winsteps (version 3.63.02) [Computer Software]. Chicago: Winsteps.com; 2010.

50. López-García E, Banegas JR, Pérez-Regadera AG, Gutiérrez-Fisac JL, Alonso J, Rodríguez-Artalejo F: Valores de referencia de la versión española del cuestionario de salud SF-36 en población adulta de más de 60 años. Med Clin (Barc) 2003, 120:568-573.

51. Louie GH, Ward MM: Sex disparities in self-reported physical functioning: true differences, reporting bias, or incomplete adjustment for confounding? J Am Geriatr Soc 2010, 58:1117-1122.

52. Sarkisian CA, Gruenewald TL, Boscardin WJ, Seeman TE: Preliminary evidence for subdimensions of geriatric frailty: the MacArthur study of successful aging. J Am Geriatr Soc 2008, 56:2292-2297.

53. Sourial N, Wolfson C, Bergman H, Zhu B, Karunananthan S, Quail J, Fletcher $J$, Weiss D, Bandeen-Roche K, Béland F: A correspondence analysis revealed frailty deficits aggregate and are multidimensional. $J$ Clin Epidemiol 2010, 63:647-654.

54. Hicks GE, Shardell M, Alley DE, Miller RR, Bandinelli D, Guralnik JM, Lauretani F, Simonsick EM, Ferrucci L: Absolute strength and loss of strength as predictors of mobility decline in older adults: the InCHIANTI study. J Gerontol A Biol Sci Med Sci 2012, 67:66-73.

55. Wolfe F, Michaud K, Pincus T: Development and validation of the health assessment questionnaire II: a revised version of the health assessment questionnaire. Arthritis Rheum 2004, 50:3296-3305.

56. Bruce B, Fries JF, Ambrosini D, Lingala B, Gandek B, Rose M, Ware JE Jr: Better assessment of physical function: item improvement is neglected but essential. Arthritis Res Ther 2009, 11:R191.

57. Chachamovich E, Fleck MP, Power M: Literacy affected ability to adequately discriminate among categories in multipoint Likert Scales. J Clin Epidemiol 2009, 62:37-46.

doi:10.1186/1477-7525-10-147

Cite this article as: Cabrero-García et al:: Validation of a mobility item bank for older patients in primary care. Health and Quality of Life Outcomes 2012 10:147.

\section{Submit your next manuscript to BioMed Central and take full advantage of:}

- Convenient online submission

- Thorough peer review

- No space constraints or color figure charges

- Immediate publication on acceptance

- Inclusion in PubMed, CAS, Scopus and Google Scholar

- Research which is freely available for redistribution 